Muro de la Investigación, 2018: 3(1), Enero-Julio, ISSN: 2523-2886

DOI:https://doi.org/10.17162/rmi.v3i1.1110

\title{
Gestión escolar y compromiso organizacional en el logro de aprendizajes de educación Intercultural Bilingüe en el nivel primario
}

\author{
Edgar Luis Martínez Huamán ${ }^{1 \mathrm{a}}$ y Salomón Vásquez Villanueva ${ }^{2}$ \\ Universidad Nacional José María Arguedas, Perú123 \\ Universidad Peruana Unión, Peru²
}

Recibido: 17 de junio de 2018

Aceptado: 03 de diciembre de 2018

Resumen

La investigación tuvo como objetivo analizar los resultados cualitativos que permiten explicar los resultados cuantitativos sobre gestión escolar y compromiso organizacional en el logro de aprendizajes en las instituciones de educación primaria: Educación Intercultural Bilingüe de la provincia de Andahuaylas. El estudio se enmarca dentro de la investigación mixta. Para la parte cuantitativa, se utilizó el cuestionario de gestión escolar adaptado de la matriz de evaluación de la calidad de la gestión de Instituciones de educación básica regular cuyo prueba de fiabilidad de Alfa de Cronbach fue de ,988; Respecto al compromiso organizacional se utilizó el cuestionario propuesto por Meyer y Allen cuya prueba de fiabilidad fue de ,987; para logros de aprendizaje se utilizó los resultados de la Evaluación Censal de Estudiantes (ECE) de cuarto grado de primaria de Educación Intercultural Bilingüe (EIB) en lectura, tanto en lengua originaria como en castellano como segunda lengua del Ministerio de Educación. La población para la parte cuantitativa consideró a 54 instituciones educativas del nivel de educación primaria que aplican la Educación Intercultural Bilingüe en la provincia de Andahuaylas, 459 estudiantes y 67 docentes. Para la parte cualitativa se consideró seis docentes, tres directores y un coordinador. Los resultados obtenidos permiten demostrar que existe relación significativa positiva entre gestión escolar y compromiso organizacional siendo la correlación de Spearman de 0,897**. Sobre gestión escolar, las instituciones educativas se encuentran ubicadas en el nivel de poco avance y los resultados sobre logros de aprendizaje se encuentran en el nivel en proceso

Palabras clave: Actitudes Lingüísticas, el Idioma quechua, sociolingüística Andina, el quechua en la universidad

\footnotetext{
${ }^{a}$ Correspondencia al autor

E-mail: emartinezh@upeu.edu.pe
} 


\title{
School management and organizational commitment in the achievement of Intercultural
}

\author{
Bilingual education learning at the primary level
}

\begin{abstract}
The objective of the research was to analyze the qualitative results that explain the quantitative results of school management and organizational commitment in the achievement of learning in Primary Education institutions: Bilingual Intercultural Education in the province of Andahuaylas, Peru. The study uses mixed research. For the quantitative part, the questionnaire of adapted school management of the Matrix of Evaluation of the quality of the management of institutions of regular Basic Education was used. This instrument has a Cronbach's Alpha value of .988. Regarding the organizational commitment, the questionnaire proposed by Meyer and Allen was used, whose reliability test was .987. For Learning Achievement, the results of the Census Student Assessment (ECE) of the fourth grade of Bilingual Intercultural Education (EIB) in reading were used, both in the original language and in Spanish as a second language from the Ministry of Education. The population for the quantitative part considered 54 educational institutions from the level of primary education that apply Intercultural Bilingual Education in the province of Andahuaylas, with a total of 459 students and 67 teachers. For the qualitative part, six teachers, three directors and one coordinator were considered. The results obtained show that there is a significant positive relationship between school management and organizational commitment, with Spearman's correlation of $0.887 * *$. Regarding school management, educational institutions are located at the level of little progress and the results on learning achievements are at the level of in process.
\end{abstract}

Key-words: Linguistic Attitudes, Quechua Language, Andean Sociolinguistics, Quechua in the University

\section{Introducción}

Los actuales sistemas educativos en la mayoría de los países consideran a la gestión escolar un tema prioritario de su agenda por lo vienen desarrollando esfuerzos conjuntos para determinar si la educación que se imparte es de calidad. Frente a la preocupación mundial por la calidad de la educación existen instituciones como la United Nations Educational Scientific and Cultural Organization (UNESCO); el Instituto Internacional para la Educación Superior en la América Latina y el Caribe (IESALC); entre otros, los cuales plantean la necesidad de abarcar realidades más amplias y realizar análisis comparativo de políticas y sistemas educativos relacionados con la calidad educativa. Sin embargo, no se puede hablar de calidad educativa sino se considera a la gestión escolar como uno de los factores claves para mejorar el logro de los aprendizajes y de esta manera alcanzar la calidad educativa. Por eso, de acuerdo con el Instituto Peruano de Evaluación, Acreditación y Certificación de la Calidad de la Educación Básica (IPEBA, 2012) la gestión 
escolar es la "capacidad que tiene la institución para dirigir sus procesos, recursos y toma de decisiones, en función a la mejora permanente del proceso de enseñanza-aprendizaje y el logro de la formación integral de todos los estudiantes que atiende” (pág. 11).

La literatura especializada considera que una gestión escolar eficiente asegura un buen logro del desempeño académico de los estudiantes, pero también necesita de una serie de factores como una eficiente planificación, un buen clima de aprendizaje, una gestión curricular adecuada, recursos humanos y financieros disponibles, entre otros. En las instituciones educativas, a nivel nacional y local, se observa como problemática, una dirección escolar centrada en el aspecto administrativo y sin metas concretas sobre el logro de aprendizaje de los estudiantes, un descuido del liderazgo por parte de los directivos, así como una carencia de diálogo y comunicación con la comunidad educativa, con los padres de familia quienes se encuentran alejados de la escuela, y en muchos casos, no prestan apoyo en la formación de sus hijos. Esto se agrava cuando se observa el poco compromiso organizacional de directivos y docentes por mejorar los procesos educativos y resultados académicos de los estudiantes reflejados en la Evaluación Censal traducidos en Logros de Aprendizaje que se realiza cada año en nuestro país. Los resultados de la Evaluación Censal de Estudiantes que presenta el Ministerio de Educación sobre el Logro de Aprendizaje de los estudiantes muestran que la región Apurímac está ubicada entre los últimos lugares cuyos resultados en lectura se encuentran en inicio $(10,7 \%)$, en proceso $(53,1 \%)$ y satisfactorio $(36,2 \%)$. Los resultados en Matemáticas señalan que se encuentran en inicio (41,5\%), en proceso (40,9\%) y satisfactorio $(17,6 \%)$.

Toda esta problemática se presenta dentro de un contexto lingüístico en las confluyen lenguas y culturas, donde el quechua sigue siendo la lengua predominante y la ruralidad viene siendo desplazada por el crecimiento de la ciudad. Por ello, se viene desarrollando la Educación Intercultural Bilingüe como una propuesta pedagógica que toma en cuenta la diversidad cultural y lingüística de los pueblos originarios para desarrollar procesos de aprendizaje de manera contextualizada a partir de sus saberes, conocimientos y valores, donde el abordaje y el desarrollo de la lengua materna y la segunda lengua de los estudiantes, tanto en el aula como en la comunidad se dan desde una mirada reflexiva y crítica de la situación sociolingüística. Una escuela intercultural bilingüe es "aquella que brinda un servicio educativo de calidad a niños, niñas y adolescentes de inicial, primaria y secundaria que pertenecen a un pueblo indígena u originario, y que hablan una lengua originaria como primera o como segunda lengua” (Ministerio de Educación, 
2013). En la provincia de Andahuaylas se viene impulsando la educación intercultural bilingüe y la generalización del quechua para todos como un aspecto pertinente a la realidad socio cultural y lingüística de la zona. Si bien la Educación Intercultural Bilingüe (EIB) está orientada a la población originaria que tiene una lengua distinta al castellano, la Educación Intercultural (EI) que se propone para todos, considera también el aprendizaje de manera voluntaria de una lengua originaria como segunda lengua por parte de la población no indígena. En efecto, la Educación Intercultural busca promover en el resto de la población peruana el interés y deseo de aprender una lengua originaria de nuestro país no sólo como un elemento simbólico de identidad nacional, sino porque muchas de estas lenguas originarias pueden y deben alcanzar un desarrollo que les permita ampliar su uso a distintos ámbitos del quehacer académico, laboral y cultural nacional.

Por otro lado, la participación de los integrantes de la comunidad educativa en la gestión escolar es de vital importancia tal como señalan Lavin (2007), Cantón (2007), Martínez (1995), Uribe (2005), entre otros. Por eso, como señala Amarante (2000), cuando se participa en una estructura organizativa, se produce la asunción personal o grupal de una determinada cuota de poder que no debe estar más que al servicio de los intereses comunes y objetivos institucionales, prescindiendo de los intereses individuales. La gestión educativa, no implica simplemente el uso de las herramientas administrativas en la conducción de la institución, por eso Gairin (1994) señala que tiene varios componentes como son los aspectos administrativos y pedagógicos y también debemos tener en cuenta que en esta labor no solamente intervienen los actores contratados explícitamente para esta función, sino que implica la participación de los diferentes agentes de la comunidad educativa, entre ellos, los padres de familia. La gestión escolar en particular, para Cantón (2007) implica “impulsar la conducción de la institución hacia determinadas metas a partir de una planificación, para lo que resultan necesarios saberes, habilidades y experiencias respecto del medio sobre el que se pretende operar" (p. 78); así como sobre las prácticas y mecanismos utilizados por las personas implicadas en las tareas institucionales como es señalado por Topete (2001), Elizondo et al (2001), Furlan (1995). Para una construcción de la escuela y del aula como un espacio destinado al logro de los aprendizajes de los estudiantes según Conde (2011) se requiere de una gestión escolar participativa, comprometida, colegiada y flexible, entendiendo gestión escolar como el conjunto de acciones que se realizan en la escuela para organizar y administrar su funcionamiento, lo cual incluye la manera como se toman decisiones y se resuelven los conflictos, señalado por Alvariño (2000). 
Para que una gestión escolar sea eficiente y eficaz se requiere además de un alto nivel de compromiso organizacional de todos los integrantes de la comunidad educativa. Por ello, en el compromiso organizacional debe existir algún tipo de esfuerzo, algo más que identificación por parte del trabajador. Por ello, el compromiso organizacional no solamente es la satisfacción personal respecto al trabajo que realiza el individuo, sino la implicación del trabajo que realiza en beneficio de la propia organización. Según Bayona y Goñi (2007) para que exista una eficacia organizacional deberá depender, en gran medida, de que los trabajadores logren conseguir y alcanzar los objetivos institucionales. Esto depende, de los recursos humanos, así como de la estructura organizativa como lo planteado por Chiavenato (2004). Aspecto esencial e importante es el recurso humano con que cuenta la organización. El compromiso organizacional, según la de Meyer y Allen (1991) tiene como componentes: el compromiso de continuidad, el compromiso afectivo y el compromiso normativo.

Asimismo, la Evaluación Censal de Estudiantes (ECE) es una evaluación estandarizada que anualmente realiza el Ministerio de Educación, a través de la Oficina de Medición de la Calidad de los Aprendizajes (UMC), para saber qué y cuánto están aprendiendo los estudiantes de escuelas públicas y privadas del país. Estas evaluaciones se realizan en el nivel primario y secundario en las áreas de matemáticas, comprensión lectora, etc. En las instituciones educativas con enfoque intercultural bilingüe las evaluaciones comprenden lectura en lengua originaria y lectura en castellano como segunda lengua. Los resultados se presentan de la siguiente manera: a) en inicio cuando el estudiante no logró los aprendizajes esperados para el ciclo; en proceso cuando el estudiante solo logró parcialmente los aprendizajes esperados al finalizar el ciclo y satisfactorio cuando el estudiante logró los aprendizajes esperados para el ciclo. La Evaluación Censal de Estudiantes.

El presente trabajo, en la que se utilizó un enfoque de investigación mixta, tuvo como objetivo general: analizar los resultados cualitativos que permiten explicar los resultados cuantitativos sobre gestión escolar y compromiso organizacional en el logro de aprendizajes en las instituciones de Educación Primaria: Educación Intercultural Bilingüe de la provincia de Andahuaylas. Asimismo, los objetivos de la investigación cuantitativa fueron determinar la relación entre gestión escolar y compromiso organizacional, entre gestión escolar y entre compromiso organizacional y logro de aprendizajes en las instituciones de Educación Primaria: Educación Intercultural Bilingüe de la provincia de Andahuaylas. Los objetivos de la investigación 
cualitativa fueron describir y caracterizar la gestión escolar; explicar de qué manera se evidencia el compromiso organizacional y explicar los resultados del logro de aprendizajes de los estudiantes de las instituciones de Educación Primaria: Educación Intercultural Bilingüe de la provincia de Andahuaylas.

\section{Metodología}

Considerando la naturaleza del objeto de estudio, así como la utilización de distintas técnicas de recolección de información se ha creído conveniente la combinación del enfoque cuantitativo y cualitativo en la investigación. Por tanto, en esta investigación se ha utilizado el método mixto de investigación, porque la metodología de la investigación mixta es una forma de investigación en la que los investigadores combinan técnicas, métodos, conceptos de tipo cuantitativo y cualitativo en una misma investigación (Fernández y otros, 2014).

\section{Participantes}

Los participantes para la parte cuantitativa fueron 459 estudiantes y 67 docentes de 54 instituciones educativas del nivel de educación primaria que tienen como enfoque la Educación Intercultural Bilingüe en la provincia de Andahuaylas. Para la parte cualitativa se consideró seis docentes, tres directores y un coordinador de Acompañamiento de Soporte Pedagógico Intercultural.

Instrumentos

En la parte cuantitativa, el cuestionario utilizado para gestión escolar, fume adaptado de la Matriz de Evaluación de la calidad de la gestión de Instituciones de Educación Básica regular (Actualización versión 2.0) que comprende 60 ítems y cuya prueba de fiabilidad de Alfa de Cronbach fue de 988 ; el cuestionario de compromiso organizacional propuesto por Meyer y Allen consta de 21 ítems y cuya prueba de fiabilidad fue de ,987; para Logros de aprendizaje se utilizaron los resultados de la Evaluación Censal de Estudiantes del año 2016, correspondiente a cuarto grado de primaria de Educación Intercultural Bilingüe (EIB) en lectura, tanto en lengua originaria como en castellano como segunda lengua.

Análisis de datos 
Los resultados fueron procesados por el paquete estadístico SPSS 24 y R como software estadístico. Para la obtención de la fiabilidad del instrumento se utilizó la correlación de Spearman para la viariables cuantitativas

\section{Resultados}

Lengua materna de los estudiantes

\subsection{Resultados de la parte cuantitativa}

Los resultados encontrados fueron los siguientes:

En la tabla 1 se observa los resultados sobre gestión escolar. Se aprecia que el 52,2\% de instituciones educativas se encuentran en un nivel de poco avance; el 31,3\% se encuentran en un nivel de Inicio y un 16,4\% se encuentran en un nivel de avance significativo. Ninguna institución educativa se encuentra ubicada en el nivel Logrado. Estos resultados anteriores reflejan la situación actual de las instituciones educativas respecto a la gestión porque muchas de ellas no tienen las condiciones necesarias y favorables para realizar una gestión educativa de calidad.

Tabla 1

Resultado de Gestión escolar en las instituciones de educación primaria: Educación Intercultural Bilingüe, Andahuaylas, 2016.

\begin{tabular}{lrrrrr}
\hline \multirow{2}{*}{$\begin{array}{l}\text { Gestión } \\
\text { Escolar }\end{array}$} & $\begin{array}{c}\text { En } \\
\text { inicio }\end{array}$ & $\begin{array}{c}\text { Poco } \\
\text { avance }\end{array}$ & $\begin{array}{c}\text { Avance } \\
\text { significativo }\end{array}$ & Logrado & Nivel de logro \\
\hline Frecuencia & 21 & 35 & 11 & 0 & Poco avance \\
Porcentaje & 31,3 & 52,2 & 16,4 & 0 & \\
\hline
\end{tabular}

En la tabla 2 se aprecian los resultados para la variable compromiso organizacional. Se observa que el 38,8\% se encuentran en un nivel moderado; el 34,3\% se encuentran en un nivel débil y un $26,9 \%$ se encuentran en un nivel fuerte de compromiso organizacional. En conclusión, respecto al compromiso organizacional el nivel en que se encuentran los docentes es moderado.

\section{Tabla 2}


Resultado de la variable Compromiso organizacional en las instituciones de educación primaria: Educación Intercultural Bilingüe, Andahuaylas, 2016

\begin{tabular}{lrrrl}
\hline \multicolumn{1}{c}{ Compromiso } & \multicolumn{3}{c}{ Categorías } & \multirow{2}{*}{ Nivel } \\
\cline { 2 - 4 } \multicolumn{1}{c}{ organizacional } & Débil & Moderado & Fuerte & \\
\hline Frecuencia & 23 & 26 & 18 & \multirow{2}{*}{ Moderado } \\
Porcentaje & 34,3 & 38,8 & 26,9 & \\
\hline
\end{tabular}

Sobre los resultados de Logros de Aprendizaje, específicamente en Lectura en lengua originaria en el distrito de Andahuaylas, en la Unidad de Gestión Educativa Local (UGEL) de Andahuaylas los resultados fueron: en Inicio 32,0\%; en proceso 57,7\% y Satisfactorio el 10,3\%; la medida promedio fue de 495. En la UGEL de Huancarama los resultados fueron: en Inicio 42,9\%; en proceso 45,0\% y Satisfactorio el 12,1\%; la medida promedio fue de 481.

Tabla 3

Resultado de la provincia de Andahuaylas en Lectura en lengua originaria en las instituciones de educación primaria: Educación Intercultural Bilingüe, Andahuaylas, 2016

\begin{tabular}{|c|c|c|c|c|}
\hline \multirow{2}{*}{$\begin{array}{l}\text { Lectura en Lengua } \\
\text { originaria }\end{array}$} & \multicolumn{3}{|c|}{ Nivel de logro } & \multirow{2}{*}{ Med. Prom } \\
\hline & En inicio & En proceso & Satisfactorio & \\
\hline UGEL Andahuaylas \% & 32,0 & 57,7 & 10,3 & En proc. \\
\hline UGEL Huancarama \% & 42,9 & 45,0 & 12,1 & 481 En proc. \\
\hline
\end{tabular}

Respecto a los resultados obtenidos en la Región Apurímac, en el año 2016, en castellano como segunda lengua, se observa que el 39,3\% se encuentra ubicado en el nivel de logro en proceso; un $27,3 \%$ se encuentra en el nivel En inicio y un 33,3\% se encuentra en el nivel de Satisfactorio. La medida promedio fue de 576 puntos lo que le ubica en el nivel de En proceso.

Tabla 4

Resultado regional de Lectura en castellano como segunda lengua en las instituciones de educación primaria: Educación Intercultural Bilingüe, Andahuaylas, 2016

\begin{tabular}{lrrcc}
\hline Lectura en & \multicolumn{3}{c}{ Nivel de logro } & Med. Prom \\
\cline { 2 - 4 } $\begin{array}{c}\text { castellano como } \\
\text { segunda lengua }\end{array}$ & En inicio & En proceso & Satisfactorio & 576 \\
\hline Porcentaje & 27,3 & 39,3 & 33,3 & En proceso \\
\hline
\end{tabular}




\subsection{Resultados de la parte cualitativa}

Una vez obtenido los resultados cuantitativos y cualitativos para poder completar la investigación mixta se realizó la triangulación de los datos cuantitativos y cualitativos.

Existe una concordancia respecto a los resultados sobre gestión escolar. El nivel de logro de las instituciones educativas es de Poco avance; esto se puede explicar porque el Estado no tiene alcance en zonas rurales, se aprecia la falta de una infraestructura adecuada, del apoyo con materiales educativos, de mecanismos de mayor control sobre los resultados de la gestión escolar. Asimismo, se tiene dificultades sobre la gestión curricular respecto a la planificación curricular, el uso de estrategias para ambas lenguas; asimismo se aprecia que el currículo considera parcialmente la historia, los conocimientos y valores de la cultura local. Por todo ello, el nivel de logro obtenido en el cuestionario es de Poco avance.

Los resultados sobre compromiso organizacional señalan que el nivel es moderado. Es decir, el nivel de compromiso no es alto ni es débil. Sin embargo, en la entrevista también se observa esta tendencia moderada; sin embargo, en la mayoría de respuestas de la entrevista señalan que el compromiso es alto.

Los resultados de la Evaluación Censal de Estudiantes de cuarto grado de primaria de Educación Intercultural Bilingüe están ubicados en nivel de Proceso, es decir, los estudiantes lograron parcialmente los aprendizajes esperados para el IV ciclo. Los estudiantes se encuentran en camino de lograrlo, pero todavía tienen dificultades. En los resultados de la entrevista, al haberse seleccionado dos instituciones educativas de cada nivel de logro los resultados cualitativos también difieren en función al nivel de logro. Sin embargo, se concluye en base a los resultados proporcionados por el Ministerio de Educación en las que se aprecia avances significativos, una mejora en los resultados de la evaluación censal.

\section{Conclusión}

Se concluye que existe relación significativa positiva entre gestión escolar y compromiso organizacional en las instituciones de Educación Primaria: Educación Intercultural Bilingüe de la

provincia de Andahuaylas. La correlación de Spearman fue de $0,897^{* *}$ lo que indica que hay correlación positiva muy alta, esto implica que a mejor gestión escolar mejor compromiso 
organizacional en las instituciones de Educación Primaria: Educación Intercultural Bilingüe de la provincia de Andahuaylas.

Asimismo, existe relación significativa positiva entre gestión escolar y Logros de aprendizaje en las instituciones de Educación Primaria: Educación Intercultural Bilingüe de la provincia de Andahuaylas. La correlación de Spearman fue de $0,884^{* *}$ lo que indica que hay correlación positiva muy alta, esto implica que a mejor gestión escolar mejor resultado en Logros de aprendizaje en las instituciones de Educación Primaria: Educación Intercultural Bilingüe de la provincia de Andahuaylas.

Por otro lado, existe relación significativa positiva entre compromiso organizacional y Logros de aprendizaje en las instituciones de Educación Primaria: Educación Intercultural Bilingüe de la provincia de Andahuaylas. La correlación de Spearman es $0,906^{* *}$ lo que indica que hay correlación positiva muy alta, esto implica que a mejor compromiso organizacional mejor resultado en Logros de aprendizaje en las instituciones de Educación Primaria: Educación Intercultural Bilingüe de la provincia de Andahuaylas.

\section{Referencias}

Alvariño, C. et al. (2000). Gestión Escolar. Un estado del arte de la literatura. Santiago: Fundación Chile.

Amarante, A. (2000). Gestión directiva. Módulos de perfeccionamiento docente 1 al 4. Buenos Aires: Magisterio del Río de la Plata.

Bayona, C. y Goñi S. (2007). Compromiso organizacional: implicaciones para la gestión estratégica de los recursos humanos. Recuperado de www.upna.es

Cantón, j. (2000). Las organizaciones escolares: hacia nuevos modelos. Buenos Aires: FUNDE.

Conde, S. (2001). Propuestas para construir una gestión escolar democrática. México: DECEyEC-IFE/Mimeo

Chiavenato, I. (2004). Comportamiento Organizacional. México DF: Thompson

Elizondo, A. (Coord.) (2001). La nueva escuela I, Dirección, liderazgo y gestión escolar. México: Paidós

Gairin, J. (1994). Organización en los centros educativos. Aspectos básicos. Barcelona. Páxis 
Furlán, A. (1995). Gestión y desarrollo institucional en Procesos curriculares institucionales y organizacionales. México: COMIE

Hernández, R., Fernández, C., \& Baptista, L. (2014). Metodología de la investigación (Sexta ed.). México: Mc Graw Hill Educación.

IIPE- UNESCO (1999). La formación de recursos humanos para la gestión educativa en América Latina. Buenos Aires: IIPE- UNESCO.

IPEBA (2012). ¿Qué y cómo evaluamos la gestión de la institución educativa? Matriz y Guía de autoevaluación de la gestión educativa de instituciones de educación básica regular. Perú: Instituto Peruano de Evaluación, Acreditación y Certificación de la Calidad de la Educación Básica (IPEBA).

Lagomarsino R. (s-f). Compromiso Organizacional. Revista de Antiguos Alumnos de la Universidad de Montevideo, pp. 79-83.

Lavín, S. (2007). Transitando desde la gestión de un establecimiento hacia la gestión de un centro de desarrollo educativo. Educare, (2), 23-35

Martínez, M. (1995). La gestión escolar como quehacer escolar. México: UPN

Meyer, J. y Allen, N. (1991): A three component conceptualization of Organizational Commitment. Human Resource Manegement Review, Vol. 01, pp. 61-89.

Ministerio de Educación. (2013). Hacia una Educación Intercultural Bilingüe de Calidad. Propuesta Pedagógica. Lima-Perú: Minedu

Pozner, P. (1997). El directivo como gestor de aprendizajes escolares. Buenos Aires, Argentina: AIQUE.

Topete, C. (2001). Desafíos y políticas de formación para la gestión en educación media superior. En Memoria VI Congreso Nacional de Investigación Educativa. Universidad de Colima, Manzanillo, 6-10 de noviembre de 2001

UMC (2006). Comprendiendo la escuela desde su realidad cotidiana. Estudio cualitativo en cinco escuela estatales de Lima”. Unidad de Medición de la Calidad Educativa. Perú: Ministerio de Educación.

Uribe, M. (2005). El liderazgo docente en la construcción de la cultura escolar de calidad. Un desafío de orden superior. Revista PRELAC, 1, 107-115.

Vasilachis, I. (2006). Estrategias de investigación cualitativa. España: Gedisa Editorial. 
Zamora, G. (2009). Organizational Commitment of chilean teachers and theirs relationship to intention to remain in their schools. Revista Latinoamericana de Psicología, Vol. 41, № 03, pp. 445-460. 